\title{
Г.Е. Кедрова
}

\section{Профессионально-ориентированные коммуникативные сервисы Веб 2.0 как основа научно-образовательной виртуальной среды нового типа}

(на примере специализированных профессиональных социальных сетей)

В статье проанализированы предпосылки и условия формирования и распространения специализированных профессионально-ориентированных социальных сетей как одного из наиболее значимых для развития современной научно-образовательной сферы компонентов Веб 2.0. Показано, как профессиональные социальные сети могут поменять всю концепцию современного образования и стать основой нового формата виртуальной образовательной среды (на примере научно-образовательных социальных сетей ResearchGate, Mendeley, Academia.edu).

Ключевые слова: Веб 2.0, социальные сети, профессиональные социальные сети, управление знаниями, виртуальная образовательная среда.

Интенсивное развитие и совершенствование технологий и сервисов Интернета, в первую очередь коммуникационных возможностей Веб 2.0, привело к вовлечению в активное информационное взаимодействие посредством Сети значительной части населения земного шара. На фоне этой глобальной тенденции симптоматично, что в десятку наиболее популярных в мире интернет-сайтов, помимо площадки электронной торговли Amazon и таких крупных поисковых систем, как Google и Baidu, входят также информационные продукты коллективного формирования знаний и управления информационными потоками. К ним относятся: интернет-энциклопедия Wikipedia, социальные сети Facebook и Twitter, многофункциональные (т. е. объединяющие возможности индивидуализированного поиска, информационного обмена и коллективного формирования информационных пространств) информационные порталы Qq.com и Yahoo, социальный видеосервис

(C) Кедрова Г.Е., 2016 
Youtube (табл.). Эти данные свидетельствуют о существенно возросшей роли программных средств глобализации информационного обмена, поэтому важно понимать, что Глобальная сеть может и должна сегодня рассматриваться как особый механизм системы управления знаниями как в локальном, так и в глобальном масштабе.

В настоящий момент вопросы, связанные с осмыслением социальных последствий феномена сетевой коммуникации, который за очень короткое время превратился в явление глобального характера, стали одними из самых актуальных для современного информационного общества. Очевидно, что они выдвинулись на первый план в результате нового, довольно специфического этапа развития технологий Интернета, который получил название Веб 2.0. Своеобразным «полигоном» для апробации разрабатываемых технологий и наиболее часто ассоциируемым с «новым» форматом Интернета сервисом стала сфера социального сетевого взаимодействия (social networking) и реализуемые на этой базе разные типы социальных сетей (social networks).

Таблища

Наиболее популярные интернет-ресурсы по данным аналитической службы http://www.alexa.com/topsites (первые десять мест по состоянию на апрель 2016 г.)

\begin{tabular}{|c|c|}
\hline Интернет-ресурс & Характеристика \\
\hline Google.com & $\begin{array}{l}\text { Глобальный интернет-сервис, обеспечивающий универ- } \\
\text { сальный информационный поиск, а также предоставляю- } \\
\text { щий доступ к наиболее популярным облачным сервисам }\end{array}$ \\
\hline Youtube.com & $\begin{array}{l}\text { Социальный видеосервис, обеспечивающий поиск } \\
\text { видеофайлов, загрузку и обмен файлами, а также все } \\
\text { социальные сетевые базовые опции }\end{array}$ \\
\hline Facebook.com & Социальная сеть \\
\hline Baidu.com & $\begin{array}{l}\text { Китайский интернет-сервис, обеспечивающий универ- } \\
\text { сальный информационный поиск на китайском языке, а } \\
\text { также предоставляющий доступ к наиболее популярным } \\
\text { облачным сервисам }\end{array}$ \\
\hline Yahoo.com & $\begin{array}{l}\text { Интернет-портал, предоставляющий возможности касто- } \\
\text { мизированного информационного поиска, а также доступ } \\
\text { к базовым облачным сервисам }\end{array}$ \\
\hline Amazon.com & $\begin{array}{l}\text { Глобальная универсальная торговая площадка в Интер- } \\
\text { нете }\end{array}$ \\
\hline
\end{tabular}


Профессионально-ориентированные коммуникативные сервисы...

Окончание табл.

\begin{tabular}{|l|l|}
\hline Интернет-ресурс & \multicolumn{1}{|c|}{ Характеристика } \\
\hline Wikipedia.org & $\begin{array}{l}\text { Общедоступная многоязычная универсальная интер- } \\
\text { нет-энциклопедия, реализованная на принципах Wiki. } \\
\text { В настоящий момент - наиболее популярный и самый } \\
\text { крупный справочный информационный ресурс (по } \\
\text { данным сайта Aleха.com) }\end{array}$ \\
\hline Google.co.in & $\begin{array}{l}\text { Индийский интернет-сервис на базе Goоgle, обеспе- } \\
\text { чивающий универсальный информационный поиск, а } \\
\text { также предоставляющий доступ к наиболее популярным } \\
\text { облачным сервисам }\end{array}$ \\
\hline Twitter.com & $\begin{array}{l}\text { Социальная сеть для публичного обмена короткими } \\
\text { заметками в формате SMS через специализированный } \\
\text { Веб-интерфейс (т. н. микроблогинг) }\end{array}$ \\
\hline Qq.com & $\begin{array}{l}\text { Китайский информационный портал, предоставляющий } \\
\text { возможности кастомизированного информационного } \\
\text { поиска, а также доступ к базовым облачным сервисам }\end{array}$ \\
\hline
\end{tabular}

Как отмечали эксперты в августе 2016 г., современная аудитория одной из самых болыших социальных сетей - Facebook - уже сравнялась с численностью населения Земли, проживавшего на нашей планете 100 лет назад ${ }^{1}$. В последнее время в этой сфере также активно развивается и совершенно новое направление - специализированные профессиональные сети (professional social networks), последствия которого для мировой науки и образования только еще предстоит осознать. Однако, несмотря на общественный запрос, можно утверждать, что изучение коммуникативных особенностей сетевой функциональности пока только зарождается и не изобилует аналитическими публикациями. Поэтому в настоящей работе мы попытаемся проследить возникновение и развитие специализированных профессиональных социальных сетей, в которых, на наш взгляд, отрабатываются базовые универсальные модели формирования и управления новыми знаниями, а также проанализировать их потенциальную значимость для всей научно-образовательной сферы в целом.

В последнее время исследователи Глобальной сети повсеместно отмечают проявление тенденции по выдвижению на первый план таких видов совместной коллективной деятельности, которые направлены на формирование в процессе межличностной коммуникации некоторого нового знания, в том числе неочевидного, что рассматривается как уникальная форма использования возможно- 
стей Интернета². Поэтому так важно изучать сегодня постоянную эволюцию разных способов информационного наполнения Сети, среди которых наиболее важным представляется исследование наиболее востребованных форм социальной коммуникации. К ним относятся разного рода «облачные» сервисы, фольксономии и вики-сайты, мультимедийные базы знаний, сетевые онтологии, семантические и социальные сети и другие аналогичные формы сетевой активности. Отметим также, что на этой основе в последнее время активно развиваются разные инновационные образовательные проекты по формированию и управлению знаниями, в частности массовые онлайновые учебные курсы (МОOС), специализированные профессиональные социальные сети и т. п.

Хотя появление специальных программных средств поддержки сетевой коммуникации и их внедрение в повседневную практику взаимодействия в Интернете массовых пользователей произошло достаточно давно (прообраз современных социальных сетей - Six Degrees.com - появился в 1997 г., а первая по-настоящему интернациональная социальная сеть LiveJournal - в 1999 г. ${ }^{3}$ ), до сих пор в современном обществе не выработано общего подхода к оценке социальной значимости этого феномена и не проведено его теоретического осмысления. Поэтому неудивительно, что само понятие вызывает неоднозначную и зачастую диаметрально противоположную общественную реакцию - от призывов немедленного внедрения формата сетевого социального взаимодействия во все сферы общественных коммуникативных практик до полного запрета на государственном уровне использования некоторых (или даже всех) видов социальных сетей. Заметим, что последнее, в частности, характерно, прежде всего, для тех обществ, в которых социальные сети рассматриваются как СМИ нового типа, основной целью которых является формирование особых «коммуникативных структур» ${ }^{4}$. Как пишет А.А. Калмыков:

Это [сервисы WEB 2.0. - Г. К.] скорее новая идеология, а не технология. Речь, следовательно, должна идти не о возможностях разнообразных сетевых сервисов, а о восприятии их аудиторией и взращивании на этой широко и быстро осваиваемой коммуникативной почве новых форм социальности и, возможно, политического поведения 5 .

К настоящему моменту опубликовано несколько полномасштабных исследований целого ряда наиболее распространенных в мире социальных сетей. В частности, одной из наиболее изученных с точки зрения механизмов функционирования, информаци- 
онного наполнения, поведенческих стратегий и социально-психологических последствий для пользователей стала социальная сеть Facebook как наиболее глобальная и самая многочисленная (по корпоративным данным, за март 2016 г. более 1 млн пользователей ежедневно обращаются к этой социальной сети, 84,2 \% всех ее пользователей проживают вне США и Канады $\left.{ }^{6}\right)$. В последнее время появились также исследования по национально ориентированным социальным сетям, в частности русскоязычной сети ВКонтакте, южнокорейской сети CyWorld, а также по некоторым другим подобным интернет-проектам и др. ${ }^{7}$ Для наиболее крупных и масштабных сетей были сформулированы основные статистические закономерности их формирования (напр. возможность/невозможность применения «теории шести рукопожатий») и определены базовые математические модели функционирования (в частности, адаптированная теория безмасштабных графов). На основе полученных данных были предложены методики использования возможностей социальных сетей в СМИ и рекламе, в сфере образования, в медицине, социальной работе, политической и идеологической сфере ${ }^{8}$. Возможные отрицательные последствия чрезмерного увлечения использованием социальных сетей для психологического и физического состояния пользователя также не были обойдены вниманием ${ }^{9}$.

Несмотря на определенный скептицизм по отношению к социальным сетям, практически все исследователи отмечают их уникальные возможности, в первую очередь, для управления информационными потоками и формирования нового знания, что нашло отражение в попытках представления специализированных социальных сетей как некоторого материализованного аналога «коллективного разума», характеризующего определенное сообщество ${ }^{10}$. Поэтому в самое последнее время в центр внимания и пользователей, и исследователей феномена социальных сетей переместились специализированные профессиональные сетевые сообщества и тематические социальные сетевые проекты, ориентированные на сферу образования и науки, в том числе такие, которые способны обеспечивать постоянно идущий и быстрый обмен результатами научных исследований ${ }^{11}$.

Нам представляется, что для возникновения и беспрецедентно быстрого и широкого распространения специализированных профессионально-ориентированных социальных сетей необходимо было формирование целого ряда специальных предпосылок и условий. Мы считаем, что основные этапы, предшествовавшие расцвету глобальных специализированных социальных сетей, на 
которых были заложены основы их функциональности, связаны с появлением следующих сервисов Глобальной сети:

1) создание и распространение средств онлайновой индексации (bookmarking) материалов, размещаемых в Интернете (в первую очередь, научных и образовательных ресурсов), и связанная с этим практика использования «облачных» сервисов;

2) выход на массового пользователя масштабных проектов по формированию научных и учебных электронных онлайновых интернет-библиотек;

3) создание программных «облачных» приложений для формирования и поддержания референциальных баз данных (персонифицированных профессиональных каталогов индексируемых библиотечных ресурсов);

4) освоение массовым интернет-пользователем средств социального сетевого взаимодействия в применении к персональным библиографическим онлайновым каталогам: предоставление управляемого доступа к индексированным библиотечным ресурсам, обмен ссылками, а также коллективное их обсуждение (комментирование и аннотирование) самыми широкими кругами профессионально-ориентированных сообществ.

Рассмотрим более подробно каждый из вышеперечисленных факторов.

1. Онлайновая индексачии интернет-ресурсов и обмен ссылками. Одним из первых программных средств онлайновой индексации материалов Интернета можно считать онлайновый интернет-сервис Delicious (первоначальное название del.icio.us, 2003 г.), немного позже появился аналогичный сервис Diigo (2006 г.). Оба сервиса бесплатно предоставляли зарегистрированным пользователям возможность составления персональных каталогов аннотированных «закладок» на любые понравившиеся сайты Интернета (internet-bookmarking), а также эффективные средства обмена своими «закладками» с другими пользователями Сети (т. н. social bookmarking).

Интерес к такого рода программным средствам в профессионально-ориентированных сообществах возник сразу же после их предъявления пользователям Глобальной сети. В первую очередь, новый сервис осваивали преподаватели средней и высшей школы, а также разработчики специализированных образовательных программ для бизнес-сообщества и программ повышения квалификации ${ }^{12}$. Используя образовательный потенциал технологий формирования «социальных закладок», в разных странах на этой базе начали появляться прообразы специализированных учебных соци- 
альных сетей с обширными хранилищами специально отобранных образовательных ресурсов, снабженных метаданными (авторскими «тэгами»), которые совместно создавали как сами педагоги, так и специалисты в области информации.

Таким образом, дистанционное обучение и компьютерная дидактика оказались той передовой областью, в которой специфические «облачные» технологии и новые программные средства и технологии «социальных закладок» начинают играть ключевую роль. На этой основе активно развивались другие программные приложения для распределенной коллективной работы, которые позволяли формировать, сохранять и поддерживать в актуализированном состоянии различные базы данных, в частности онлайновые тематические собрания ссылок на учебно-научные материалы.

Параллельно рассмотренным тенденциям и инновациям в Интернете стремительно расширяется сфера использования технологий блогинга (программных средств, позволяющих любому пользователю создавать и поддерживать в актуальном состоянии т. н. «интернет-дневник событий», а также комментировать публикации других авторов-блогеров). Поэтому можно утверждать, что на данном этапе ключевая роль в социализации накопленной с помощью механизма социальных закладок информации постепенно переходит к блогам, которые тем самым становятся своеобразной «школой» по распространению новых знаний, а также специальных знаний, умений и навыков цивилизованного социального интернет-взаимодействия (т. е. стандартов «электронного профессионализма», или “e-professionalism”). На основе практики блогинга в ряде профессионально-ориентированных сфер начинают формироваться локальные виртуальные сообщества, входящие в глобальную блогосферу, в которых отрабатываются специфические навыки коллективной обработки информации. Впоследствии эти навыки и умения легли в основу функционирования практически всех видов новых социальных сетевых сервисов, в том числе и профессиональных социальных сетей. Важно, что в соединении с технологиями социальных сетей, позволяющими комментировать и снабжать тэгами собранные ссылки, на базе коллективных и персональных библиотек-репозиториев достаточно быстро начинают формироваться разные варианты специализированных информационных гипертекстовых пространств, которые можно рассматривать как один из возможных вариантов виртуальной образовательной среды $^{13}$. В этой области неоценимую помощь оказали традиционные библиотечные правила и нормативы для аннотирования и реферирования информации, в первую очередь научно-образовательной, 
а также разработанные в этой сфере универсальные международные системы библиографического описания. Впоследствии этот многолетний опыт также нашел отражение в теоретических и практических исследованиях, посвященных особенностям формирования единой универсальной компьютерной системы метаданных, которую можно было бы применить к любому виду информационного продукта ${ }^{14}$.

2. Интернет-библиотеки как предпосылка формирования спещиализированных профессиональных социальных сетей. На базе разрабатываемых алгоритмов распределенной переработки информационных потоков достаточно быстро оказались востребованными новые пакетные сервисы, позволяющие издательствам и библиотекам реализовывать масштабные проекты по информационному обеспечению поиска и сохранения информации об имеющихся и появляющихся научных публикациях и изданиях. Эти сервисы обеспечивали каталогизацию и автоматическое реферирование и аннотирование в распределенных онлайновых электронных интернет-библиотеках всей хранящейся в них информации независимо от формы ее представления (традиционной печатной или цифровой, текстовой или мульти- и гипермедийной и т. п.). Пионером в этой области выступил ведущий американский научный институт Institute of Scientific Information (ISI), в котором с конца 1950-х гг. изучаются тенденции развития научного знания и разрабатываются новые принципы наукометрии. На основе разработок этого института была создана специализированная поисковая интернет-платформа "Web of Science / Web of Knowledge”, объединившая реферативные базы данных публикаций в научных журналах (всего около 10 тыс. наиболее авторитетных и востребованных научных журналов), базы данных патентов, собрание монографий, материалов конференций и публикации в научных сборниках.

Наиболее важной частью платформенных сервисов WoS стал учет взаимного цитирования всех публикаций, входящих в охватываемые этой платформой базы данных. Этот показатель стал одним из наиболее востребованных для изучения тенденций развития и управления научным процессом информационных ресурсов, поэтому основным продуктом такого рода платформ (и впоследствии большинства цифровых электронных библиотек) стал автоматизированный мониторинг «индексов цитирования», самым известным из которых на сегодняшний день является индекс Хирша (h-index). Благодаря этой информации такие библиографические базы данных сегодня очень активно привлекаются также органами государственного планирования и организации науки и образования. 
В них любой зарегистрированный пользователь достаточно оперативно и просто может получить интегрированную информацию по целому ряду параметров оценки эффективности научной работы. В частности, система позволяет генерировать отчеты о публикационной активности - научной производительности отдельных ученых, научных коллективов и организаций, об эффективности сложившихся международных научных коллективов, целесообразности планируемых научных коллабораций, о востребованности отдельных научных публикаций, влиятельности и авторитетности каждого ученого, работы которого представлены в базе данных. Общая оценка ученого представлена его рейтингом, вычисляемым на основе цитируемости работ исследователя, нормированной в зависимости от медианных показателей, отражающих типы и скорость информационного обмена в разных областях научного знания.

Впоследствии аналогичные поисковые интернет-платформы начали разрабатывать самые крупные и авторитетные мировые научные издательства (Elsevier, Springer, Wiley и др.), а также национальные и транснациональные специализированные коммерческие компании - аггрегаторы научной информации (JSTOR, MUSE, EBSCO и т. п.). Вслед за ними целый ряд университетских издательств и национальных и международных компаний и фондов инициировали масштабные электронные библиотечные онлайновые проекты (например, Cambridge University Press, Oxford University Press, Taylor \& Francis, а также аналогичный российский проект Российской научной электронной библиотеки - eLibrary). Актуальность всех подобных проектов потребовала выработки рекомендаций по формам и способам эффективной интеграции научных электронных онлайновых библиотек в современные учебные программы по использованию ИКТ в вузовском и послевузовском образовании.

3. Социальные сервисы в онлайновых научно-образовательных интернет-библиотеках. Быстрое и повсеместное распространение практики социальных закладок вместе с внедрением в процессы переработки информации библиотечных аналитических интернет-сервисов подготовило мировое научное сообщество к освоению новых универсальных программных решений. Эти решения обеспечили объединение в едином интернет-пространстве практик социальных закладок, технологии аннотирования и комментирования публикаций в системах социальной коммуникации (блогах), работу в персональных онлайновых библиотечных ресурсах с типовыми интернет-сервисами, формирующими пространство социальных сетей. 
Одной из первых электронных онлайновых персональных библиотек такого типа стал проект Mendeley, разработанный учеными ряда университетов Великобритании в 2007 г. и приобретенный в 2013 г. Издательским домом Elsevier. Проект Mendeley (www. mendeley.com) предоставляет зарегистрированным пользователям, которыми могут быть представители образовательных учреждений любого уровня, находить, читать и каталогизировать в собственной «облачной» библиотеке заинтересовавшие их научно-образовательные ресурсы (статьи, проекты, неопубликованные рабочие материалы и т. п.), вести по каждому из ресурсов совместную работу с коллегами через предоставление коллективного доступа к отдельным статьям и материалам, экспортировать и импортировать данные из других электронных библиотек и репозиториев и формировать на основе своей библиотеки пристатейные списки использованной литературы, а также создавать свой профиль ученого, публиковать результаты собственных исследований, комментировать и обсуждать чужие научные публикации, размещать в своем профиле отзывы и рекомендации, задавать вопросы и отвечать на вопросы, публикуемые другими членами Mendeley-сообщества. Проект Mendeley оказался очень успешным в среде ученых и преподавателей, а также в среде студентов и аспирантов высших учебных заведений, его сообщество очень быстро расширялось и по состоянию на начало 2016 г. в его сети уже было зарегистрировано 4,6 млн пользователей. Вслед за успехом проекта Mendeley другая крупнейшая издательская и аналитическая корпорация Thomson Reuters также представила аналогичную платформу EndNote, которая, в отличие от Mendeley, является полностью коммерческим продуктом и, возможно, поэтому развивается не столь успешно.

4. Специализированные профессиональные социальные сети. Возникновение специализированных узкопрофессиональных социальных сетевых сервисов принято связывать с появлением сети LinkedIn (2003 г.), которая изначально позиционировала себя как специализированная социальная сеть для представителей бизнес-сообщества. За ней последовал целый ряд аналогичных проектов, предназначенных для самых разных целевых аудиторий (педагогов, физиков и химиков, историков и т. п.). Хотя большинство таких проектов не получило развития в дальнейшем, однако специализированные социальные сети, целью которых являлся обмен научными публикациями, распространение научной информации и - в идеале - формирование нового знания, напротив, оказались очень успешными. Этот факт вполне соответствует предсказаниям одного из наиболее авторитетных «гуру» в области Веб 2.0 Криса 
Андерсона, который утверждал, что будущее Интернета связано не с появлением все новых и новых гигантских социальных сетей, а с тем, что мировому сообществу требуется бесконечное число микросоциальных сетей, которые будут специализироваться на отдельных тематических областях ${ }^{15}$. Феномен специализированных социальных сетей для ученых оказался настолько важным для академического сообщества, что ему даже была посвящена специальная статья в наиболее авторитетном в современном мире журнале "Nature"16.

Сегодня к таким научным социальным сетям, которые приобрели достаточно глобальные масштабы, относят: ResearchGate (9 млн. пользователей), Academia.edu (более 6 млн. участников) и социальный блок сети Mendeley (4,6 млн. пользователей) ${ }^{17}$. Эти три основные профессиональные научно-образовательные социальные сети принято называть «большой тройкой» - "big three", формирующей костяк всего современного научного информационного пространства. Рассмотрим подробнее некоторые особенности использования и коммуникативные возможности этих сетевых социальных профессионально-ориентированных проектов.

Научная социальная сеть ResearchGate (2008 г.) - проект, с самого начала создаваемый под лозунгом «учеными для ученых», объединяет социальные сетевые сервисы с развитыми средствами обмена электронными референциальными базами данных научных публикаций, персональными динамическими рейтингами пользователей и рекрутинговыми информационными ресурсами. Одной из наиболее востребованных опций социальной сети ResearchGate является возможность задать вопрос и самому опубликовать ответ на любой другой вопрос, заданный пользователями сети, на общей ленте «вопросов-ответов». Эта функция сети является наиболее популярной и по данным опроса, проведенного журналом "Nature" 18 . Отмечают ее особую значимость и российские участники проекта, в частности в дискуссиях российских блогеров можно встретить утверждения, что самое «крутое» в ResearchGate - это «участвовать в обсуждении того или иного вопроса учеными из разных стран и учреждений, особенно если вопрос междисциплинарный, весьма увлекательно, и главное полезно - либо узнаешь что-то новое, будь то фактология или свежий взгляд на вопрос, во-вторых, если встреваешь в дискуссию, то нет-нет приходится с полки достать запылившийся учебник и освежить былое. <..> Лично мне гораздо интереснее сидеть там, чем в Контакте...» (Пост Антона Алипова на интернет-форуме ФИРАН $)^{19}$. 
Другой важной и пока уникальной особенностью проекта ResearchGate является возможность загрузить в свой профиль не только опубликованные научные статьи, автором/соавтором которых является пользователь (в том числе, препринты), но и статьи, которые по разным причинам не попали в печать, а также разноформатные исследовательские материалы и даже негативные данные экспериментов, которые также представляют большой интерес для работающих в этой области ученых. В 2009 г. ResearchGate предоставил пользователям возможность загружать недавно опубликованные статьи с полным соблюдением авторских прав.

Одной из отличительных особенностей проекта ResearchGate являются разработанные его создателями специальные программные средства семантического поиска, который позволяют заново проиндексировать не только ключевые слова, но и аннотации всех информационных ресурсов социальной сети а также материалов других ведущих интернет-репозиториев научных публикаций (PubMed, CiteSeer и т. п.). На основе найденных семантических соответствий пользователям предлагается возможность нахождения и установления новых контактов с другими участниками социальной сети, занимающимися близкими по тематике вопросами. Все эти возможности выводят профессиональную социальную сеть ResearchGate на лидирующие позиции в этой сфере, что позволило одному из основателей сети Джаду Мэдишу (Ijad Madisch) утверждать, что она «изменит весь научный процесс таким образом, который невозможно полностью предугадать» ("With ResearchGate we're changing science in a way that's not entirely foreseeable") ${ }^{20}$.

Другой крупный проект специализированной научной социальной сети - Academia.edu (2008 г.) - является также чрезвычайно популярным в современной академической среде, однако его пользователи существенно менее часто и регулярно, чем пользователи сети ResearchGate, взаимодействуют друг с другом на этой сетевой платформе ${ }^{21}$. Этот проект объединяет типичные социальные сетевые сервисы со средствами обмена научными публикациями, а также показателями востребованности и цитируемости размещенных в его базе публикаций. В отличие от большинства других специализированных профессиональных социальных сетей в проекте Academia.edu могут свободно регистрироваться любые заинтересованные лица, возможно поэтому около половины всех его участников - это аспиранты и студенты, а также так называемые независимые исследователи. Такое разнообразие в 
профессиональной принадлежности участников социальной сети позволило выявить в результате специального исследования одно из наиболее важных и фундаментальных свойств специализированных профессиональных социальных сетей, а именно то, что они, будучи полностью виртуальными сообществами, в достаточно высокой степени коррелируют с практикой информационного взаимодействия в реальных профессиональных научных сообществах $^{22}$.

Таким образом, можно констатировать, что за последние годы все базовые сервисы, которые определяют возможности и преимущества нового этапа развития Интернета - Веб 2.0 - активно проникают в повседневную профессиональную практику академического и образовательного сообщества. Помимо того что виртуальное «присутствие», как минимум, в одной из наиболее популярных специализированных профессиональных сетей постепенно становится не только желательным, но и во многих ситуациях (в частности, в сфере междисциплинарных исследований) обязательным для самопрезентации ученого / преподавателя, все большее число студентов и аспирантов постоянно обращаются к ресурсам разных специализированных социальных сетей. Мы считаем, что это делает их de facto одним из наиболее перспективных аналогов виртуальной образовательной среды нового поколения (VLE 2.0). В частности, специализированные социальные сетевые сервисы уже доказали свою эффективность на стадии формирования базовых образовательных и исследовательских знаний и навыков (умение находить и отбирать релевантную и актуальную информацию, навыки критического осмысления и анализа информации с разных точек зрения, освоение алгоритмов и технологий совместной коллективной работы, умение делиться информацией, корректно вести дискуссию и др.). Они, несомненно, окажут в самое ближайшее время решающее влияние на преобразование всей сферы образования и науки, что было отмечено еще в 2010 г. в отчете по вопросам образовательного потенциала Веб 2.0, подготовленного Европейской комиссией. В этом отчете прозвучал призыв изучать изменения и трансформации существующих образовательных моделей и организационных процессов в области науки и образования в результате массового освоения новых социальных сервисов Интернета, которые, в свою очередь, могут кардинально поменять всю систему управления научным процессом, сформировать новые алгоритмы оценки эффективности научных и учебных учреждений, изменить практику сертификации и аккредитации образовательных и научных учреждений ${ }^{23}$. 
Примечания

1 Эксперты: Аудитория Facebook сравнялась с населением Земли 100 лет назад // PLANET TODAY. 2016. 28 июля. [Электронный ресурс] URL: http://planet-today.ru/novosti/tekhnologii/item/46083-eksperty-auditoriya-facebook-sravnyalass-naseleniem-zemli-100-let-nazad (дата обращения: 30.07.2016).

2 Патаракин Е.Д., Ярмахов Б.Б. Веб 2.0 - управление, изучение и копирование // Образовательные технологии и общество. 2007. № 2. С. 245-258.

3 Boyd D., Ellison N.B. Social Network Sites: Definition History and Scholarship // Journal of Computer-mediated Communication. Vol. 13. Issue 1. 2007. P. 210-230. [Электронный ресурс] URL: https://www.researchgate.net/publication/220438020_Social_Network_Sites_Definition_History_and_Scholarship (дата обращения: 30.07.2016).

4 Калмыков А.А. Интернет-журналистика в системе СМИ: Становление, развитие, профессионализация: Автореф. дис. ... д-ра филол. наук. М., 2009.

5 Там же. С. 32.

6 Воронкин А.С. Социальные сети: эволюция, структура, анализ // Образовательные технологии и общества. 2014. Т. 17. № 1. С. 650-675.

7 Boyd D., Ellison N.B. Op. cit.; Воронкин A.C. Указ. соч.

8 Патаракин Е. Д., Ярмахов Б.Б. Веб 2.0 - управление, изучение и копирование // Образовательные технологии и общество. 2007. № 2. С. 245-258; Thibaut P. Social network sites with learning purposes: Exploring new spaces for literacy and learning in the primary classroom // Australian Journal of Language and Literacy. Vol. 38. № 2. 2015. P. 85-93.

9 Kuss D.J., Griffiths M.D. Online Social Networking and Addiction: A Review of the Psychological Literature / Int. J. Environ // Public Health. 2011. No. 8. P. 35283552; Муромцева А.В., Муромцев В.В. Проблемы информационной безопасности в социальных сообществах в сети Интернет // Вестник РГГУ. Серия «Экономика. Управление. Право». 2016. № 3(5). С. 84-92.

10 Lévy P. Collective Intelligence: Mankind's Emerging World in Cyberspace. Perseus: Cambridge, MA, 1997.

11 Bik H.M., Goldstein M.C. An introduction to social media for scientists // PLoS Biol. 11(4): [Электронный ресурс] URL: https://www.ncbi.nlm.nih.gov/pmc/articles/ PMC3635859/ (дата обращения: 30.07.2016).

12 Farwell T.M., Waters R. Exploring the use of social bookmarking technology in education: An analysis of students' experiences using a course-specific delicious // Journal of Online Learning and Teaching. 2010. T. 6. № 2. C. 398.

13 Churchill D. Social bookmarking - repository - networking: Possibilities for support of teaching and learning in higher education // Serials review. 2009. T. 35. № 3. C. $142-148$.

14 Hayman S. Folksonomies and tagging: New developments in social bookmarking // Developing and Improving Classification Schemes: Ark Group Conference. Sydney, 2007. 
15 Anderson P. What is Web 2.0? Ideas, technologies and implications for education // JISC Technology and Standards Watch. 2007. February.

16 Noorden R. Scientists and the Social Networks // Nature. 2014. Vol. 512. 14 August. P. $126-129$.

17 Matthews D. Do academic social networks share academics' interests? // Times Higher Education. [Электронный ресурс] URL: https://www.timeshighereducation. com/features/do-academic-social-networks-share-academics-interests (дата обращения: 30.07.2016).

18 Ibid.

19 Интернет-форум ФИРАН: ResearchGate - что это? Зачем это? [Электронный pecypc] URL: http://forum.lebedev.ru/viewtopic.php?p=87957\&sid=61c86fb7c6f2 047e1ec1d6881086c479\#p87957 (дата обращения: 30.07.2016).

20 Noorden R. Op. cit. Р. 126.

21 Береснева Е. Ученые и социальные сети: Материалы портала «Научная Россия». 24 ноября 2014 г. [Электронный ресурс] URL: http://scientificrussia.ru/articles/ uchenye-i-socialnye-seti (дата обращения: 30.07.2016).

22 Thetwall M., Kousha K. Academia.edu: Social Network or Academic Network? // Journal of the Association for Information Science and Technology. 2014. No. 65(4). P. 721-731.

23 Arquero J.L., Romero-Frías E. Using social network sites in Higher Education: An experience in business studies // Innovations in Education and Teaching International. 2013. Vol. 50. No. 3. P. 239. 